\title{
Assessment of the shadow caused by the human body on the personal RF dosimeters reading in multipath environments
}

\author{
Alfonso Bahillo", Rubén M. Lorenzo*, Santiago Mazuelas", \\ Patricia Fernández* and Evaristo J. Abril* \\ *CEDETEL (Centre for the Development of Telecommunications) \\ *University of Valladolid \\ Spain
}

\section{Introduction}

Over the last decades, the environmental levels of electromagnetic emissions have increased due to the exponential growth rate of radio frequency (RF) systems. This fact is the cause of the concerns expressed by some members of the public about the safety of exposure to RF radiation from wireless communication devices, with particular reference to cellular phone handsets. As a consequence, several national and international organizations have set limits for human exposure to RF fields. These include the IEEE C95.1 standard (IEEE C95.1-2005, 2006) and the recommendations of the National Council on Radiation Protection and Measurements (NCRP) (NCRP, 1986), the International Commission on Non-Ionizing Radiation Protection (ICNIRP) (ICNIRP, 1999) and the National Radiation Protection Board (NRPB) in the United Kingdom (NRPB, 1993). The different standards vary somewhat in their exposure limits and in other particulars. However, at frequencies used for common wireless communication systems, these different guidelines are broadly similar. To date, despite a considerable amount of speculation, the only health effect from RF radiation identified by the scientific community has been related to an increase in body temperature. The levels of RF exposure from base stations and local wireless networks are so below the recommended limits in those guidelines that temperature increases are insignificant and they do not affect human health. An open issue for the scientific community is the possibility of long-term non-thermal effects of RF fields from which the importance of RF exposure assessment emerges.

Electromagnetic dosimetry which attempts to evaluate the interaction between electromagnetic waves and biological tissues is primarily used for the evaluation of human exposures. It has become common for exposure situations to be characterized by a mixture of spectral contributions from different services, while exposures to single frequencies are rare. This is the particular case of frequency selective dosimeters, which can measure different frequency bands while identifying the contribution of each service. Current personal dosimeters are inherently measuring devices for free field since they are calibrated 
in free space. In (Blas et al. 2007), it has been shown that personal dosimeters used to assess exposure to RF electric fields were subject to potential errors associated with perturbations of the fields caused by the presence of the human body. In a common worst-case scenario, when the dosimeter is in a shadow area generated by the presence of the human body, an underestimation up to $30 \mathrm{~dB}$ of the E-field level could occur. In (Blas et al. 2007), only waves impinging on the human front have been considered. Because of diffractions and reflections, the RF signal could impinge on the human body with different directions of arrival (DoA) and at a given location, the RF signal is the sum of various waves having different amplitudes and DoAs. Therefore, the goal of this chapter is to help in the task of assessing the human exposure by analyzing the local interaction behavior of the dosimeter with an anatomical model of the human body (Spitzer, et al. 1996) bearing in mind the multiple waves that impinges on the body with different amplitudes and DoAs in a multipath environment and at different frequencies.

As a first approach, the potential errors associated with perturbations of the fields by the presence of the human body have been evaluated taking into account that only the main Efield contribution impinges on the human body with a certain DoA and at different frequencies (Bahillo et al. 2008b). This has been made bearing in mind a single bounce multipath in addition to the direct path between the transmitter and the human body as the dominant mode of propagation. Once the effect of the E-field perturbation around the human body has been evaluated for each DoA and frequency, multiple E-field contributions impinging on the human body at the same time with different amplitudes and DoAs will be analyzed according to the different mean azimuth power distributions taking place in different multipath environments (Bahillo et al. 2008a). Due to the particular public concerns about cellular phones, this chapter focuses the analysis on the Global System for Mobile Communications frequency band (GSM-900MHz).

Computer simulations give a detailed description of the surface E-field on the human body. Nowadays, the finite-difference time-domain (FDTD) method (Taflove, 2005) is the most used technique in dosimetry studies. This algorithm is currently the most acceptable choice when a digital anatomical model of a human body has to be analyzed. Therefore, numerical simulations have been performed with the FDTD method in order to analyze the interaction between a plane wave and the whole human body in a stable situation. Forasmuch as the FDTD method is not computationally efficient to study scattering problems involving large areas such as the urban environments, the ray-tracing technique is used for the computation of the power distribution with respect to the azimuth angle averaged over a distance corresponding to 6 minutes walk, in order to evaluate the average human exposure in an urban environment.

\section{Methods and Model}

\subsection{Numerical Methods of Analysis}

In dosimetry studies, the FDTD method is currently the most acceptable choice if a digital anatomical model of a human body is going to be analyzed. Forasmuch as the FDTD method is not computationally efficient to study scattering problems involving large regions, such as urban environments, the ray-tracing technique is used for the evaluation of the azimuth angle power distribution averaged over a distance corresponding to 6 minutes walk in an urban environment. Therefore, numerical simulations are performed with the 
FDTD method to evaluate the interaction between the E-field and the human body, while the ray-tracing technique is used to perform the azimuth angle power distribution. As the FDTD method and the ray-tracing technique are well known and they are described in detail in (Taflove, 2005) and (Glassner, 1991) respectively, only a brief outline of the implementation used is described in this chapter. Due to the huge memory and computational time requirements needed, mainly by the FDTD method but also by the raytracing technique, a server with a 64-bit architecture and 16GB RAM is used to be able to tackle, in a reasonable time, the simulation model and areas.

As a first step, the FDTD method is used to study the exposure of an anatomical model of the human body according to different DoAs. A monochromatic plane wave with vertical polarization is used as excitation source. To calculate the spatial distribution of the E-field around the human body, the impulse response of the system is evaluated in the frequency domain. A narrow Gaussian pulse is considered which if it is narrow enough it would be a good approximation to an impulse. Keeping in mind the computational resources limitation, the sampling nodes must be taken to ensure that a suitable representation of the human body is made. On the other hand, a good rule of thumb is to take at least 10 points per wavelength (Taflove, 2005). To accomplish these two objectives, the grid computation cell has dimensions $2 \mathrm{~mm} \times 2 \mathrm{~mm} \times 2 \mathrm{~mm}$ resulting a good accuracy for the FDTD simulations, where the tissue in each node is assigned permittivity and conductivity values according to the frequency (Gabriel, 1996). As E-field is shared out at each half spatial increment, a threedimensional E-field is obtained every $\Delta x=4 \mathrm{~mm}$. Once the spatial increment size is chosen, and in order to avoid numerical instabilities, the time step is fixed to $\Delta t=\Delta x / 2 \mathrm{c}$ which satisfies the well-known Courant condition of scheme stability (Kunz, 1993). In order to avoid the E-field to be reflected in the limits of the computational volume, 15-layers of uniaxial perfectly matched layer (UPML) absorbing boundary conditions have been used. In addition, a total/scatterd field formulation is chosen to minimize the load on the absorbing boundary conditions and to avoid any interaction between the incident propagation wave and the absorbing boundary conditions (Sullivan, 2000).

As a second step, the two-dimensional ray tracing technique is used to evaluate the mean azimuth power distribution of the E-field in urban environments, starting from an omnidirectional base-station antenna and taking into account wall reflections and corner diffraction present in the environment. For microcellular studies wall transmission is often ignored. The ray-tracing technique is based on geometrical optics and it has been modeled with only third-order reflections and first-order diffractions. In the microcellular environment less than $50 \%$ of the incident power is reflected by the walls and even less power is diffracted by the corners. The mean azimuth power distribution is evaluated by using the "ray-launching" approach, having characterized each wall through its permittivity and conductivity values, and having used the well-known reflection and diffraction coefficients (Balanis, 1989).

\subsection{Model Description of the Human Body}

To study the interaction of the radiated E-field with an exposed subject, a full 3D human body with $2 \mathrm{~mm}$ resolution has been used for simulation purposes (see left hand of Fig. 1). This model has been obtained from tissue-classified version of the "Visible Human Project" developed at Brooks Air Force base Laboratories and sponsored by the National Library of Medicine (NLM) in the USA. The original model has $1 \mathrm{~mm}$ resolution, it is based on 
anatomical slices from a male cadaver $(1.80 \mathrm{~m}$ tall and $90 \mathrm{~kg}$ mass, adult male, aged 38$)$ and it has been downsampled to obtain a final resolution of $2 \mathrm{~mm}$. The whole human body was included inside the computational domain. The body model has a total of 31 different types of tissues/organs, for the electrical characterization of the tissues at the frequencies considered the data reported in (Gabriel, 1996) has been used.
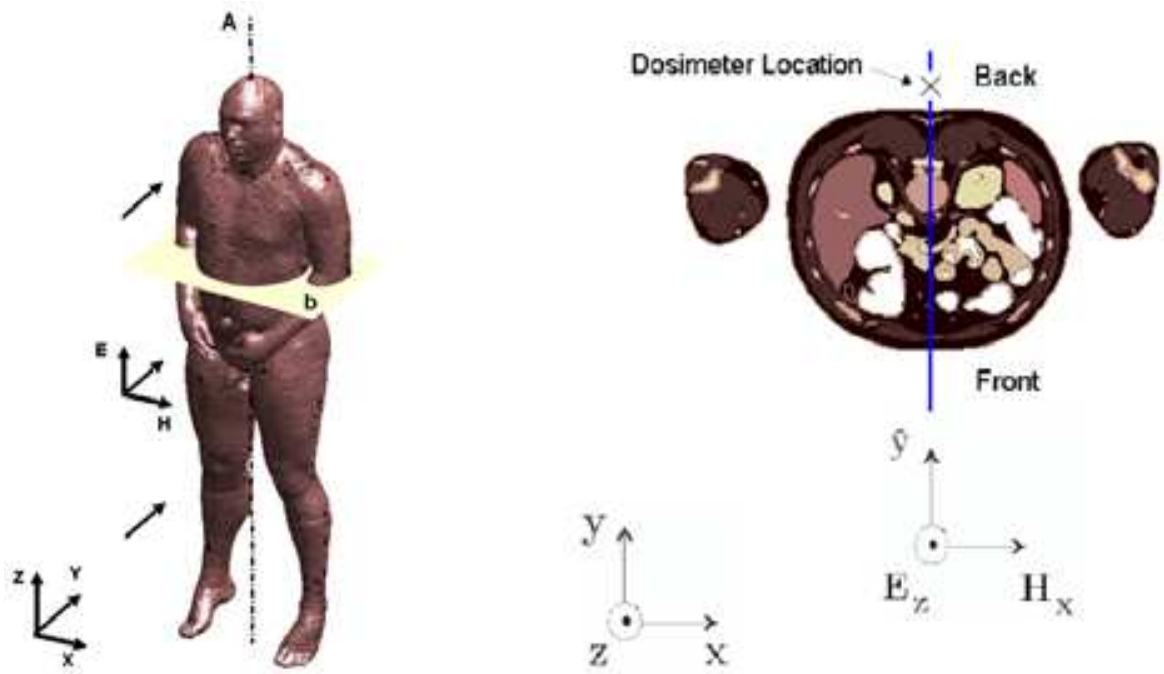

Fig. 1. Left hand, 3D human body computational volume, where $A$ is the rotation axis. Right hand, outcome visualization plane, cross-section of the anatomical human body through plane $b$ (approximately $2 / 3$ body height).

In the computational volume, $Y=0$ is the illumination plane while $Z=120 \mathrm{~cm}$ (approximately $2 / 3$ body height) is the outcome visualization plane (see right hand of Fig. 1 ). The wavefront travels toward positive values of axis $Y$ at each time step. Inside the FDTD computational domain, the presence of the human body in dosimeter reading is only considered. In order to have more control over the E-field behavior around the human body, the alteration of the pattern of the plane wave propagation has been analyzed by means of its DoA variations at different frequencies. The DoA is modified every 10 degrees from 0 to 180 degrees in azimuth. Instead of modifying the wavefront phase which illuminates the human body model, the human model has been rotated upon itself. In this way, the different scenarios have been shown as far as the DoA changes are concerned.

\section{Simulation Results}

\subsection{RF Exposure of the Human Model at Different DoAs and Frequencies}

This section presents an analysis of the surface E-field on the human body model when it is illuminated by a plane wave at different DoAs (from 0 to 180 every 10 degrees in azimuth) and frequencies (FM-100 MHz, GSM-900 MHz and DCS-1800 MHz) based on the FDTD simulations. Fig. 2 shows the simulation results. These results represent the spatial variability of the E-Field when it is compared with the incident wave through an imaginary 
line. The characteristics of this line are the following ones: it is parallel to the ground and it goes approximately through the central part of the stomach of a human $(2 / 3$ body height and $1 / 2$ body width). The assumption which states that the plane wave arrives horizontally is valid since the following topic is fulfilled, the distance between the human body and the RF source is larger than the difference between the height of the RF source and the scatters around the human body, for more details see (Petrus, 2002).

In Fig. 2 different scenarios are shown, where the human body is illuminated by a plane wave at different frequencies and from different azimuthal angles. The simulation results fit well with the experimental measurements reported by (Blas, 2007) at FM-100 and GSM-900 bands when the DoA of the plane wave is 0 degrees in azimuth. In order for the dosimeter not to bother the human or interfere with his movements, it is assumed that the person has the dosimeter fixed to his back (five centimeters from it) approximately $2 / 3$ body height, although from Fig. 2 other distances could be analyzed. Due to the relative orientation of the human body with regard to the plane wave DoA, the dosimeter could be located either within the maximum shadow zone, within the peak or in an intermediate case of the stationary wavefront which takes place when the plane wave impinges on the human body. In addition to the previous work in (Blas, 2007), simulations in DCS-1800 band are also included.

The first interesting issue is that due to the arrival angle uncertainty, there are differences in dosimeter readings up to $35 \mathrm{~dB}$ in DCS-1800, 25dB in GSM-900 and 12dB in FM-100 bands due to the human body presence, in these two opposite scenarios, when the plane wave impinges on the human body to the front or to the back. On the other hand, as frequency increases and due to the shorter wavelength, the E-field has more difficulties in penetrating the human body. Consequently, most of the E-field behind the body comes from its going around it, not from going through it. As a result, the higher the frequency, the higher the attenuation in the region shaded by the human body. In the DCS-1800 and GSM-900 bands, it is worth pointing out that the standing wave ratio when the DoA is 180 degrees is much higher than the one when the DoA is 0 degrees, because the energy has more difficulties in going around the human body when impinging on the back than when impinging on the front, because the body front is more rounded than the body back.

\subsection{Mean Azimuth Power Distribution in Urban Environments}

In a multipath scenario, such as the urban environment, the human exposure is characterized by several waves impinging on the human body with different amplitudes and DoAs. Exposure guidelines, such as those published by (ICNIRP, 1999), require exposures to be averaged over 6 minutes to be compared with their basic restrictions. By using the two-dimensional ray-tracing technique, according to that time-averaging of exposure, the mean azimuth power distribution has been computed over two different routes of a distance of $300 \mathrm{~m}$ each, corresponding to a human walking of $50 \mathrm{~m} /$ minute for 6 minutes. The routes have been simulated by using a map of a neighbourhood of the Valladolid city center, Spain (see Fig. 3). A circle ( $0.25 \mathrm{~m}$ radius) acting as a human model, the same urban environment and the same position for the omnidirectional transmitter have been considered in all the scenarios simulated. Fig. 4 shows the mean azimuth power distribution for the two different routes, which have different line-of-sight (LOS) percentage, route A with 35\% LOS and route B with $21 \%$ LOS. 


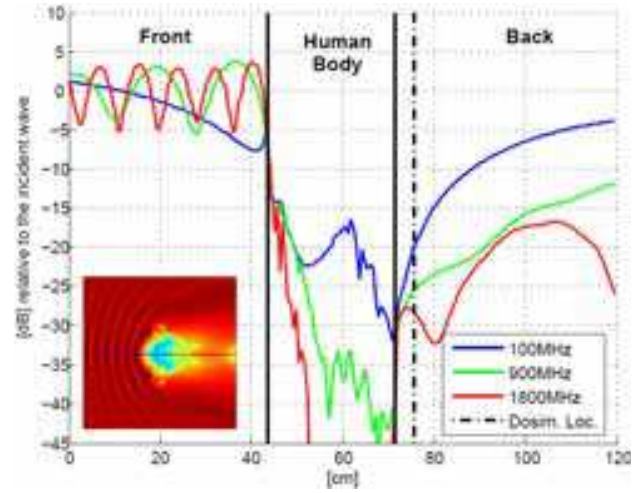

a) 0 degrees in azimuth.

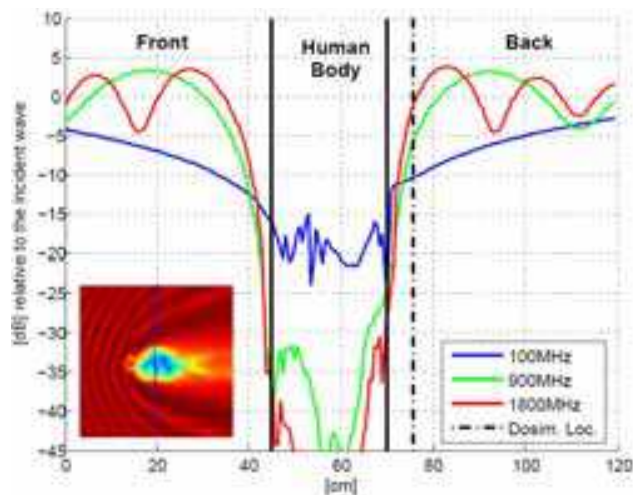

c) 90 degrees in azimuth.

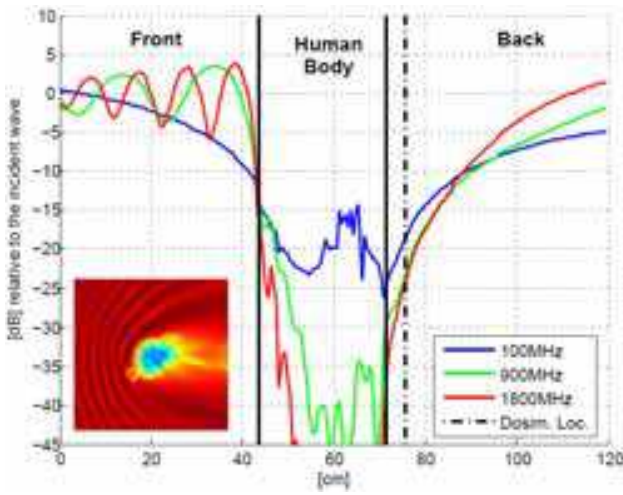

b) 45 degrees in azimuth.

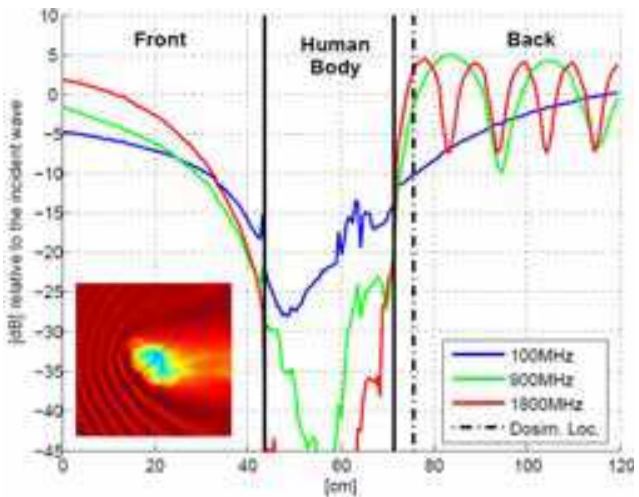

d) 135 degrees in azimuth.

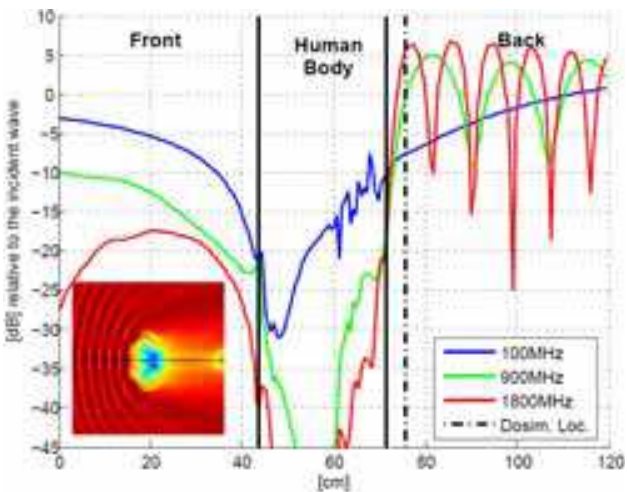

e) 180 degrees in azimuth.

Fig. 2. Spatial variability of the E-field due to the presence of the human body. Rotating the model around $A$ axis at FM-100 MHz, GSM-900 MHz and DCS-1800 MHz bands. 


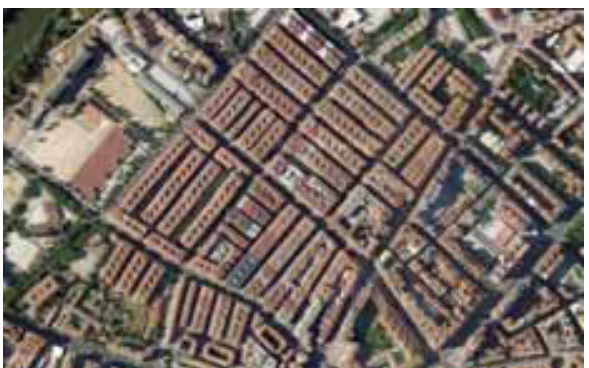

a) Valladolid city neighbourhood satellite view (by Google).

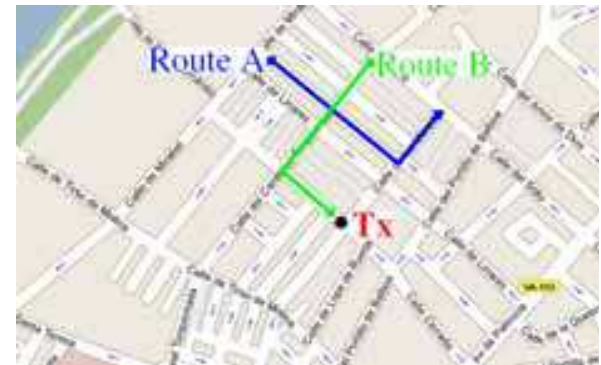

b) Valladolid city neighbourhood map view (by Google).

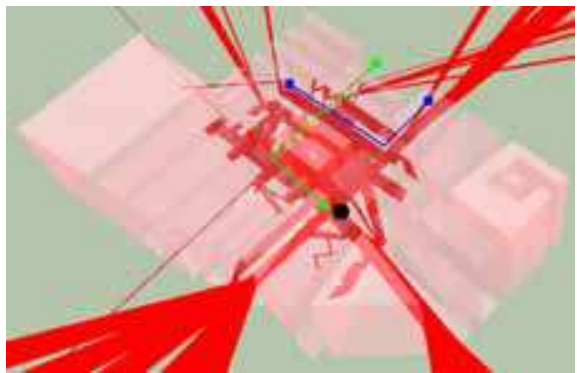

c) Ray-tracer view.

Fig. 3. Ray-tracer simulation results in the Valladolid city neighbourhood.

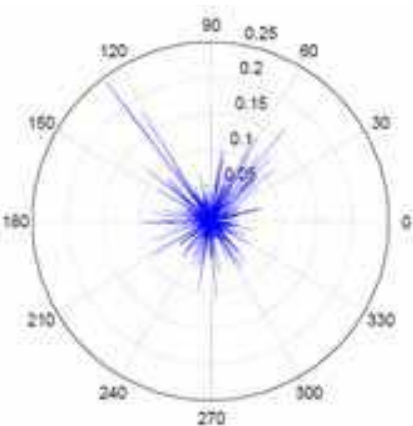

a) Route A with $35 \%$ LOS.

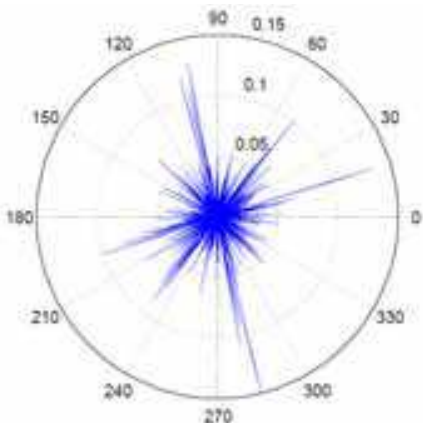

b) Route B with $21 \%$ LOS.

Fig. 4. Mean azimuth power distribution.

\section{Discussion}

In this section the effect of the E-field perturbation around the human body is going to be evaluated for each DoA and frequency taking into account that only the main E-field contribution impinges on the human body. Afterwards, multiple E-field contributions 
impinging on the human body at the same time with different amplitudes and DoAs are going to be analyzed according to the different mean azimuth power distributions which take place in different urban environments. In this case, due to the particular public concerns about the cellular phones, this section focuses on the frequency GSM-900 MHz.

\subsection{Main E-field contribution}

If a LOS path exists between the transmitter and the human body and a single bounce multipath is the dominant mode of propagation (in addition to the direct path), the multipath components will be tightly clustered in angle about the direct path component. For an urban microcell environment, it was found that the average angle spread was 9.4 degrees (Liberti, 1996). But, DoA variations of \pm 5 degrees do not change significantly the surface E-field pattern in the potential location of the dosimeter, near the human body. Under these assumptions, only the main RF azimuthal contribution would be considered. As seen in Fig. 2, the resulting shadow, solely due to the human body presence, can cause high differences in the dosimeter reading because of the DoA uncertainty of the main RF contribution, due to the relative position between the transmitter and the human body. For this reason, in order to better estimate the surface E-field behavior around the human body, it is necessary to gather information of the individual simulation results for each DoA by using an azimuthal angle with a certain probability to simulate different scenarios based on the main RF contribution. The symmetry of right and left human body is assumed.

In the first scenario, suppose that all the DoA are equally likely which takes place when the person is walking randomly in locations free from scatters or scatters have a uniform angular distribution around the subject. In the second scenario, the DoA 0 and 180 degrees are more likely than the others. This scenario takes place when the RF source is located away from a narrow street in the direction of the human front or human back equally likely. Therefore, the most probable movement of the person is the straight line. The third scenario is an intermediate situation where the street is wider than the one in the second scenario. Therefore, the person can follow other directions different from the straight line with the same probability. Table 1 presents the probability of the main contribution of the RF source of impinging with a certain DoA in these three scenarios.

\begin{tabular}{llllll}
\hline & $\mathbf{0}^{\mathbf{o}}$ & $\mathbf{4 5}^{\mathbf{0}}$ & $\mathbf{9 0}^{\mathbf{0}}$ & $\mathbf{1 3 5}^{\mathbf{0}}$ & $\mathbf{1 8 0}^{\mathbf{0}}$ \\
\hline Uni & 0.125 & $2 \times 0.125$ & $2 \times 0.125$ & $2 \times 0.125$ & 0.125 \\
NS & 0.4 & $2 \times 0.05$ & $2 \times 0$ & $2 \times 0.05$ & 0.4 \\
WS & 0.2 & $2 \times 0.125$ & $2 \times 0.05$ & $2 \times 0.125$ & 0.2 \\
\hline
\end{tabular}

Table 1. Probability of the DoA of the main E-field contribution. Uni, uniform; NS, narrow street; WS, wide street.

Fig. 5. shows the simulation results. It represents the spatial variability of the E-field compared to the incident wave caused by the presence of the human body in three different scenarios, uniform (Uni), narrow street (NS) and wide street (WS) illumination. As it can be seen in Fig. 5, five centimeters from the human back, where the dosimeter would be located, the E-field strength is similar in the three scenarios at each frequency, $-13 \mathrm{~dB}$ with a variation of $\pm 1 \mathrm{~dB}$ in FM band, $-6 \mathrm{~dB}$ with a variation of $\pm 1 \mathrm{~dB}$ in GSM- 900 band and $-3 \mathrm{~dB}$ with a variation of $\pm 1 \mathrm{~dB}$ in DCS- 1800 band. While a few centimeters from the potential 
location of the dosimeter, the E-field variation is higher. In the FM-100 band the E-field has a variation up to $2 \mathrm{~dB}$, in GSM-900 up to $12 \mathrm{~dB}$ and in DCS-1800 up to $16 \mathrm{~dB}$.

As shown in Fig. 5, under the assumption that body front and body back illumination are equally likely, the variations of the surface E-field on the body taken either from the front or from the back are the following ones: five centimeters from the body front, the E-field is approximately $2 \mathrm{~dB}$ higher than the E-field on the back at FM-100 and GSM-900 and $3 \mathrm{~dB}$ lower at DCS-1800 bands. This cannot be applied when differences of illumination probabilities occur between body front and body back.

From the results presented in Fig. 5, it can be conclude that if the dosimeter is used as a system for the assessment of personal exposures in epidemiological studies, on average, the dosimeter reading is always lower than the E-field when the human is not present.

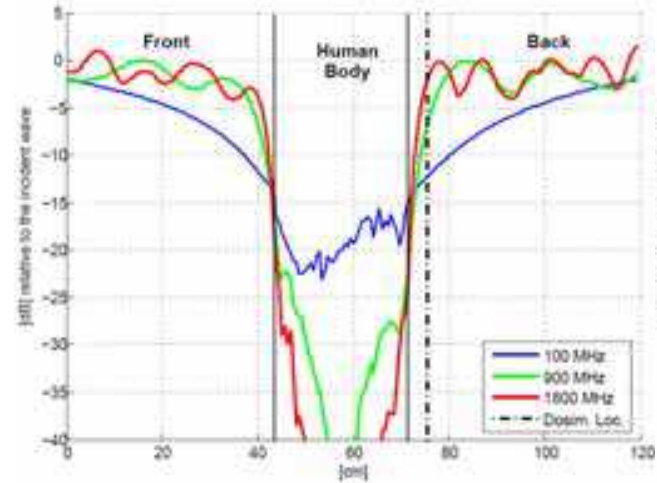

a) Uniform illumination.

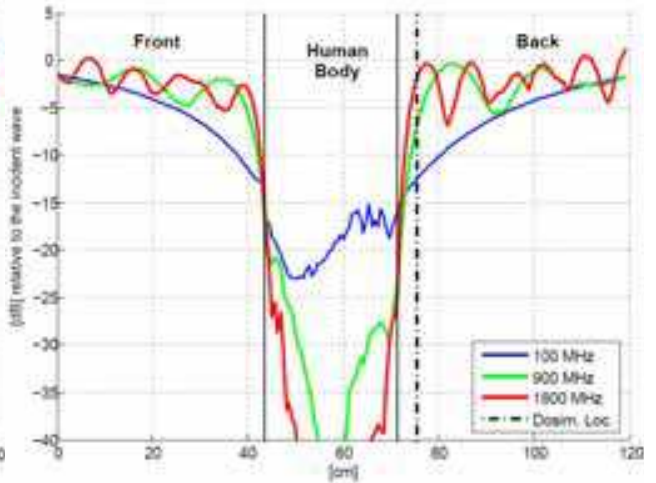

b) Narrow street illumination.

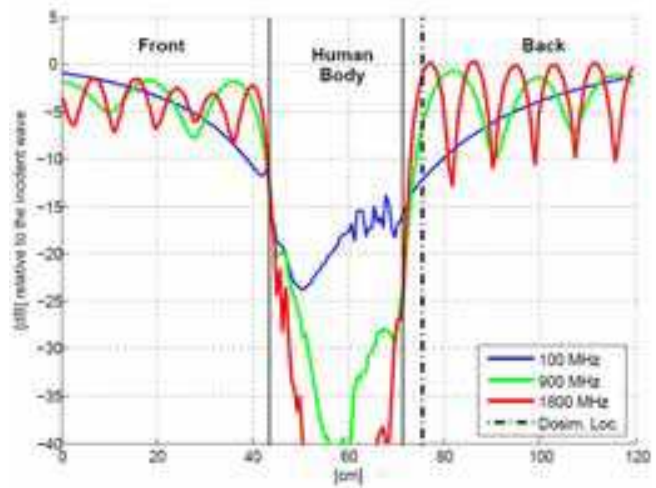

e) Wide street illumination.

Fig. 5. Spatial variability of the E-field compared to the incident wave at FM-100 MHz, GSM$900 \mathrm{MHz}$ and DCS-1800 MHz bands in three different scenarios. 


\subsection{Multiple E-field contributions}

It is natural to assume that when a mobile user moves randomly in any environment, the incident waves can arise from any azimuthal direction with equal probability. As a consequence, the uniform distribution is a justified assumption for the azimuth power distribution averaged over a random route. To better estimate the surface E-field behavior around the human body in an urban environment, four different mean azimuth power distributions have been considered, uniform distribution, routes $\mathrm{A}$ and $\mathrm{B}$ inside the neighborhood of the city of Valladolid, and the mean azimuth power distribution obtained by measurements (Kimmo, 2002) in an urban microcell in the city of Helsinki, Finland.

In simulations, the human body model is not illuminated with plane waves arising from different DoAs at the same time, but a weighted average of the individual simulation results from the different DoAs have been done. The weight of each DoA has been obtained from the mean azimuth power distribution results shown in Fig. 4 and it has been normalized to the total incident power. As a result, the different individual DoAs simulation results have been added constructively. Therefore, an upper bound is fixed under which the dosimeter reading could be found.

Fig. 6 shows the upper bound of the spatial variability of the E-field according to the four scenarios presented in this section. As it can be seen in this figure, five centimeters from the human back, where the dosimeter would be located, the E-field strength is similar for the four mean azimuth power distributions that have been considered. The dosimeter in that position would read an E-field value between $-5 \mathrm{~dB}$ and $-7.5 \mathrm{~dB}$ at GSM-900 band. Therefore, the E-field strength is underestimated even in a multipath scenario, where the dosimeter is not always located in the shadow region. A few centimeters from the potential location of the dosimeter the E-field variation is higher, in the GSM-900 band the E-field has a variation up to $6 \mathrm{~dB}$.

As it can be inferred from the results presented in Fig.6, if a body-worn dosimeter is used as a system for the assessment of personal exposures in epidemiological studies, on average, the dosimeter reading is at least $5 \mathrm{~dB}$ lower than the E-field when the human is not present. Despite of the power distribution in azimuth, no straightforward assumption can be justified for the elevation angle. It was found (Lee, 1973) that for most of the streets, field measurements of mobile radio signals have shown that signal arrival is concentrated in elevation angles lower than 16 degrees along the horizontal level. Therefore, the shadowing effect has to be analyzed also with respect to changes in elevation angles. 


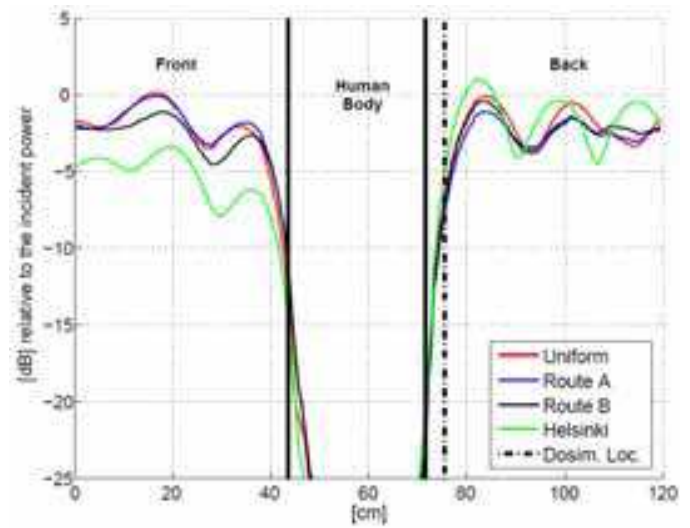

Fig. 6. Weighted average of the spatial variability of the E-field for the four different mean azimuth distributions at GSM-900 band.

\section{Conclusions}

Assuming that the dosimeter is fixed to the human body back at waist level, the first interesting issue is that due to the uncertainty in the angle of arrival of the E-field, there are differences in dosimeter readings up to $35 \mathrm{~dB}$ at DCS-1800 band, $25 \mathrm{~dB}$ at GSM-900 band and $12 \mathrm{~dB}$ at FM-100 band caused by the human body presence, in these two opposite scenarios, when the plane wave impinges on the human body to the front or to the back. On the other hand, as frequency increases and due to the shorter wavelength, the E-field has more difficulties in penetrating the human body, consequently most of the E-field behind the body comes from its going around it, not from going through it. As a result, the higher the frequency, the higher the attenuation in the area shaded by the human body. Therefore, the dosimeter reading is generally lower than the E-field strength when the human is not present. Even in a multipath scenario, where the dosimeter is not always in the shaded area, the E-field level is underestimated because, as it will be shown in this chapter, despite the differences among all the multipath scenarios analyzed, in the place where the dosimeter would be located, the variations in E-field strength are similar but always lower than the Efield when the human is not present. Therefore, understanding and predicting in which way the presence of the human body alters the plane wave propagation is a fundamental aspect when a dosimeter is used for the assessment of personal exposure in an urban environment.

\section{References}

IEEE C95.1-2005 (2006). IEEE standard for safety levels with respect to human exposure to radio frequency electromagnetic fields, $3 \mathrm{KHz}$ to $300 \mathrm{GHz}$, IEEE, ISBN: 0738148342, New York.

NCRP (1986) Biological effects and exposure criteria for radio frequency electromagnetic fields, National Council on Radiation Protection and Measurements, ISBN: 0913392804, Washington D.C. 
ICNIRP (1999) Guidelines on limiting exposure to non-ionizing radiation, International Commission on Non-Ionizing Radiation Protection, ISBN: 3-9804789-6-3, Germany.

NRPB (1993) Board statement on restrictions on human exposure to static and time-varying electromagnetic fields and radiation, Chilton Oxon NRPB, ISN: 859513661, UK.

Blas, J., Lago, F., Fernández, P., Lorenzo, R. M. and Abril, E. J. (2007). Potential exposure assessment errors associated with body-worn RF dosimeters. Bioelectromagnetics, No. 28, (July 2007) 573-576.

Bahillo, A., Blas, J., Fernández, P., Mazuelas, S., Viñuela, A., Lorenzo, R. M., and Abril, E. J. (2008a). E-Field errors associated with RF dosimeters for RF human exposure assessment in urban environments. Proceedings of $30^{\text {th }}$ annual international conference of Engineering in Medicine and Biology, pp. 2821-2824, ISBN: 978-1-4244-1814-5, Vancouver, BC, August 2008, IEEE EMBS.

Bahillo, A., Blas, J., Fernández, P., Lorenzo, R. M., Mazuelas, S. and Abril, E. J. (2008b). EField assessment errors associated with RF dosimeters for different angles of arrival. Radiation Protection Dosimetry, No. 132(1), (October 2008) 51-56.

Spitzer, V., Ackeman, M. J., Scherzinger, A. L. and Whitlock, D. (1996). The visible human male: a technical report, J. Am. Med. Inform. Assoc., No. 2, (April 1996) 118-130.

Taflove, A. (2005). Computational electrodynamics: The Finite-Difference Time-Domain method, Artech House; 3 $3^{\text {rd }}$ edition, ISBN: 1-58053-076-1, Norwood, MA.

Glassner, A. S. (1991). An Introduction to Ray Tracing, Academic Press, Harcourt Brace Jovanovich Publishers, ISBN: 0-12-286160-4, Cambridge, UK.

Balanis, C. A. (1989). Advanced Engineering Electromagnetics, John Wiley \& Sons Inc., ISBN: 047-162194-3, New York.

Gabriel, C., (1996). Compilation of the dielectric properties of body tissues at RF and microwave frequencies. Brooks Air Force, Brooks AFB, TX, Tech. Rep. AL/OE-TR1996-0037, 1996.

Kunz, K. S., and Luebbers, R. J. (1993). The Finite Difference Time Domain Method for Electromagnetics, CRC-Press, ISBN: 0-84-938657-8.

Sullivan, D. M. (2000). Electromagnetic simulation using the FDTD method. Wiley-IEEE Press, ISBN: 0-7803-4747-1, NewYork.

Petrus, P., Reed, J. H., and Rappaport, T. S. (2002). Geometrical-based statistical macrocell channel model for mobile environments. IEEE Trans. on Communications, No. 50, (2002) 495-502.

Kimmo, K., Sulonen, K., Laitinen, H., Kivekäs, O., Krogerus, J., and Vainikainen, P. (2002). Angular Power Distribution and Mean Effective Gain of Mobile Antenna in Different Propagation Environments. IEEE Trans. on Vehicular Technology, No. 51, (September 2002) 823-838.

Sacks, Z. S., Kingsland, D. M., Lee, R., and Lee, J.-F. (1995). A perfectly matched anisotropic absorber for use as an absorbing boundary condition. IEEE Trans. On Antennas Propagation, No. 43, (1995) 1460-1463.

Liberti, J. C., and Rappaport, T.S. (1996). Analysis of CDMA cellular radio systems employing adaptive antennas in multipath environments. Proceedings of $46^{\text {th }}$ international conference of Vehicular Technology, Mobile Technology for the Human Raceapos, pp. 1076-1080, April 1996, IEEE VTC.

Lee, W., and Brandt, R. H., (1973). The elevation angle of mobile radio signal arrival. IEEE Trans. On Communications, Vol. COM-21, (1973) 1194-1197. 


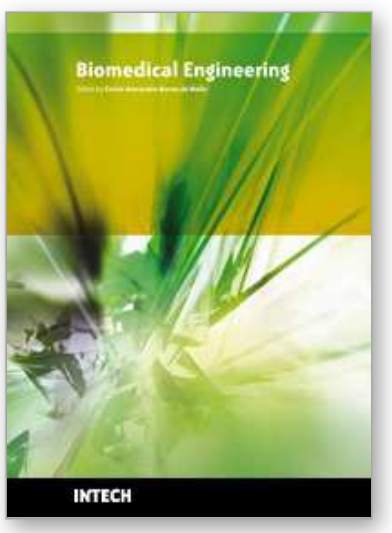

\author{
Biomedical Engineering \\ Edited by Carlos Alexandre Barros de Mello
}

ISBN 978-953-307-013-1

Hard cover, 658 pages

Publisher InTech

Published online 01, October, 2009

Published in print edition October, 2009

Biomedical Engineering can be seen as a mix of Medicine, Engineering and Science. In fact, this is a natural connection, as the most complicated engineering masterpiece is the human body. And it is exactly to help our "body machine" that Biomedical Engineering has its niche. This book brings the state-of-the-art of some of the most important current research related to Biomedical Engineering. I am very honored to be editing such a valuable book, which has contributions of a selected group of researchers describing the best of their work. Through its 36 chapters, the reader will have access to works related to ECG, image processing, sensors, artificial intelligence, and several other exciting fields.

\title{
How to reference
}

In order to correctly reference this scholarly work, feel free to copy and paste the following:

Alfonso Bahillo, Rubén M. Lorenzo, Santiago Mazuelas, Patricia Fernández and Evaristo J. Abril (2009). Assessment of the Shadow Caused by the Human Body on the Personal RF Dosimeters Reading in Multipath Environments, Biomedical Engineering, Carlos Alexandre Barros de Mello (Ed.), ISBN: 978-953-307-013-1, InTech, Available from: http://www.intechopen.com/books/biomedical-engineering/assessment-of-the-shadowcaused-by-the-human-body-on-the-personal-rf-dosimeters-reading-in-multipath

\section{INTECH}

open science | open minds

\section{InTech Europe}

University Campus STeP Ri

Slavka Krautzeka 83/A

51000 Rijeka, Croatia

Phone: +385 (51) 770447

Fax: +385 (51) 686166

www.intechopen.com

\section{InTech China}

Unit 405, Office Block, Hotel Equatorial Shanghai

No.65, Yan An Road (West), Shanghai, 200040, China 中国上海市延安西路65号上海国际贵都大饭店办公楼 405 单元 Phone: +86-21-62489820

Fax: $+86-21-62489821$ 
(C) 2009 The Author(s). Licensee IntechOpen. This chapter is distributed under the terms of the Creative Commons Attribution-NonCommercial-ShareAlike-3.0 License, which permits use, distribution and reproduction for non-commercial purposes, provided the original is properly cited and derivative works building on this content are distributed under the same license. 